\title{
Relation between Type-II Discrete Sine Transform and Type -I Discrete Hartley Transform
}

\author{
M.Narayan Murty ${ }^{1}$ and K. Sreekanth ${ }^{2}$ \\ 1,2 (Department of Physics, Raghu Institute of technology, Visakhapatnam - 531162, Andhra Pradesh, India.)
}

\section{ABSTRACT}

In this paper, a relation for finding type-II discrete sine transform (DST) from type-I discrete Hartley transform (DHT) has been derived. The transform length $N$ is taken as even. Using this relation, the $(N-1)$ output components of DST can be realized from DHT. The DHT is one of the transforms used for converting data in time domain into frequency domain using only real values.

Keywords: Discrete sine transform, discrete Hartley transform.

\section{INTRODUCTION}

Discrete transforms play a significant role in digital signal processing. Discrete cosine transform (DCT), discrete sine transform (DST) are used as key functions in many signal and image processing applications. There are eight types of DCT and DST. Of these, the DCT-II, DST-II, DCT-IV, and DST-IV have gained popularity. The DCT and DST transform of types I, II, III and IV, form a group of so-called "even" sinusoidal transforms. Much less known is group of so-called "odd" sinusoidal transforms: DCT and DST of types V, VI, VII and VIII.

The DST was first introduced to the signal processing by Jain [1], and several versions of this original DST were later developed by Kekreet al.[2], Jain [3] and Wang et al. [4]. Ever since the introduction of the first version of the DST, the different DSTs have found wide applications in several areas in Digital signal processing (DSP), such as image processing[1,5,6], adaptive digital filtering[7] and interpolation[8]. The performance of DST can be compared to that of the DCT and it may therefore be considered as a viable alternative to the DCT. For images with high correlation, the DCT yields better results; however, for images with a low correlation of coefficients, the DST yields lower bit rates [9]. Yip and Rao [10] have proven that for large sequence length $(N \geq 32)$ and low correlation coefficient $(\rho<0.6)$, the DST performs even better than the DCT.

The discrete Hartley transform (DHT) [11], [12] plays an important role in many digital signal processing (DSP) applications since it is a good alternative to the discrete Fourier transform (DFT) for its real-number operations. One of the main attractions of DHT is that it only involves real computations in contrast to complex computations in the DFT. In addition, the inverse DHT has the same form as the forward DHT, except for a scaling factor.
Therefore, a single kind of program or architecture can be used to carry out both the forward and inverse DHT computations. The DHT is one of the transforms used for converting data in time domain into frequency domain using only real values.

Over the years, the DHT has been established as a potential tool for signal processing and communication applications, e.g., computation of circular convolution, and deconvolution [13], [14], interpolation of real-valued signals [15], image compression [16], [17], error control coding [18], adaptive filtering [19], multi-carrier modulation and many other applications [20]-[22]. Fast implementation of one-dimensional (1-D DHT) has attracted many attentions [23]-[25]. However, DHT is computation intensive.

In this paper, an expression for finding DSTII from DHT-I for even transform length $N$ has been derived. The $(N-1)$ output components of DST can be realized from DHT by this method. The data flow diagram for realization DST from DHT is shown.

The rest of the paper is organized as follows. The relation between Type-II DST and Type-I DHT has been derived in Section-II. Conclusion is given in Section-III. 


\section{RELATION BETWEEN TYPE-II DST AND TYPE-I DHT}

Let $x(n), n=0,1,2, \ldots, N-1$, be the input data array. Then, the one- dimensional type - II DST can be defined as

$$
Y(k)=\sqrt{\frac{2}{N}} c_{k} \sum_{n=0}^{N-1} x(n) \sin \left[\frac{k(2 n+1) \pi}{2 N}\right] \quad \text { for } k=1,2, \ldots, N
$$

where $Y(k)$ represents the transformed data. The value of $c_{k}$ is given by

$$
c_{k}=\left\{\begin{array}{c}
1 / \sqrt{2} \text { if } k=N \\
1 \text { otherwise }
\end{array}\right.
$$

Without loss of generality, the scale factor $\sqrt{\frac{2}{N}} c_{k}$ in (1) can be omitted. After omitting the scale factor, (1) can be written as

$$
Y(k)=\sum_{n=0}^{N-1} x(n) \sin \left[\frac{k(2 n+1) \pi}{2 N}\right] \quad \text { for } k=1,2, \ldots, N
$$

The one-dimensional type-1 DHT of input sequence $\{u(n): n=0,1,2, \ldots, N-1\}$ is defined as

where $\operatorname{cas} \theta=\cos \theta+\sin \theta$.

$$
H(k)=\frac{1}{\sqrt{N}} \sum_{n=0}^{N-1} u(n) \operatorname{cas}\left(\frac{2 \pi k n}{N}\right) \quad \text { for } k=0,1,2, \ldots ., N-1
$$

Ignoring the scale factor $1 / \sqrt{N}$, (3) can be written as

$$
H(k)=\sum_{n=0}^{N-1} u(n) \operatorname{cas}\left(\frac{2 \pi k n}{N}\right) \quad \text { for } k=0,1,2, \ldots, N-1
$$

Taking the transform length $N$ as even, define

$$
u(n)=\left\{\begin{array}{l}
x(2 n) \quad \text { if } n=0,1,2, \ldots ., \frac{N}{2}-1 \\
x(2 N-2 n-1) \text { if } n=\frac{N}{2}, \ldots, N-1
\end{array}\right.
$$

Substituting (5) in (4), we have

$$
\begin{aligned}
H(k)= & \sum_{n=0}^{\frac{N}{2}-1} x(2 n) \operatorname{cas}\left(\frac{2 \pi k n}{N}\right)+\sum_{n=\frac{N}{2}}^{N-1} x(2 N-2 n-1) \operatorname{cas}\left(\frac{2 \pi k n}{N}\right) \\
& \quad \text { for } k=0,1,2, \ldots, N-1
\end{aligned}
$$

Since, $2 n$ is even, the first term in RHS of (6) is written as

$$
\sum_{n=0}^{\frac{N}{2}-1} x(2 n) \operatorname{cas}\left(\frac{2 \pi k n}{N}\right)=\sum_{m \text { even }} x(m) \operatorname{cas}\left(\frac{m \pi k}{N}\right)
$$

Expanding the second term in RHS of (6), we obtain

$$
\begin{aligned}
& \sum_{n=\frac{N}{2}}^{N-1} x(2 N-2 n-1) \operatorname{cas}\left(\frac{2 \pi k n}{N}\right)= \\
& x(N-1) \operatorname{cas}(\pi k)+x(N-3) \operatorname{cas}\left(\pi k+\frac{2 \pi k}{N}\right)+\cdots+x(1) \operatorname{cas}\left(2 \pi k-\frac{2 \pi k}{N}\right) \\
& \text { for } k=0,1,2, \ldots, N-1
\end{aligned}
$$

In the first term of RHS of the above expression,

$$
\operatorname{cas}(\pi k)=\operatorname{cas}(-\pi k)
$$

The second term in RHS of (8) can be expressed as

$$
\begin{aligned}
x(N-3) \operatorname{cas}\left(\pi k+\frac{2 \pi k}{N}\right) & =x(N-3) \operatorname{cas}\left[2 \pi k-(N-2) \frac{\pi k}{N}\right] \\
& =x(N-3)\left\{\cos \left[2 \pi k-(N-2) \frac{\pi k}{N}\right]+\sin \left[2 \pi k-(N-2) \frac{\pi k}{N}\right]\right\} \\
& =x(N-3)\left\{\cos \left[(N-2) \frac{\pi k}{N}\right]-\sin \left[(N-2) \frac{\pi k}{N}\right]\right\} \\
& =x(N-3)\left\{\cos \left[-(N-2) \frac{\pi k}{N}\right]+\sin \left[-(N-2) \frac{\pi k}{N}\right]\right\} \\
& =x(N-3) \operatorname{cas}\left[-(N-2) \frac{\pi k}{N}\right]
\end{aligned}
$$

Similarly, the last term in RHS of (8) can be written as

$$
x(1) \operatorname{cas}\left(2 \pi k-\frac{2 \pi k}{N}\right)=x(1) \operatorname{cas}\left(-\frac{2 \pi k}{N}\right)
$$

Substituting (9),(10) and (11) in RHS of (8) and reversing the order of terms in RHS, we obtain 


$$
\begin{aligned}
\sum_{n=\frac{N}{2}}^{N-1} x(2 N-2 n-1) \operatorname{cas}\left(\frac{2 \pi k n}{N}\right) & =x(1) \operatorname{cas}\left(-\frac{2 \pi k}{N}\right)+\cdots+x(N-3) \operatorname{cas}\left[-(N-2) \frac{\pi k}{N}\right] \\
\text { or } \sum_{n=\frac{N}{2}}^{N-1} x(2 N-2 n-1) \operatorname{cas}\left(\frac{2 \pi k n}{N}\right) & =\sum_{m \text { odd }} x(m) \operatorname{cas}\left[-\frac{(m+1) \pi k}{N}\right]
\end{aligned}
$$

Substituting (7) and (12) in (6), we get

$$
\text { for } k=0,1,2, \ldots, N-1
$$

$$
H(k)=\sum_{m \text { even }} x(m) \operatorname{cas}\left(\frac{m \pi k}{N}\right)+\sum_{m \text { odd }} x(m) \operatorname{cas}\left[-\frac{(m+1) \pi k}{N}\right]
$$

Multiplying (13) with cas $\left(-\frac{\pi k}{2 N}\right)$, we have

$$
\begin{aligned}
H(k) \operatorname{cas}\left(-\frac{\pi k}{2 N}\right)= & \sum_{m \text { even }} x(m) \operatorname{cas}\left(\frac{m \pi k}{N}\right) \operatorname{cas}\left(-\frac{\pi k}{2 N}\right) \\
+ & \sum_{m \text { odd }} x(m) \operatorname{cas}\left[-\frac{(m+1) \pi k}{N}\right] \operatorname{cas}\left(-\frac{\pi k}{2 N}\right) \\
& \quad \text { for } k=0,1,2, \ldots, N-1
\end{aligned}
$$

Consider the identity

$$
\operatorname{cas} \theta \operatorname{cas} \emptyset=\cos (\theta-\varnothing)+\sin (\theta-\emptyset)
$$

Using (15) in RHS of (14), we obtain

$$
\begin{aligned}
H(k) \operatorname{cas}\left(-\frac{\pi k}{2 N}\right) & =\sum_{m \text { even }} x(m)\left[\cos \left(\frac{m \pi k}{N}+\frac{\pi k}{2 N}\right)+\sin \left(\frac{m \pi k}{N}+\frac{\pi k}{2 N}\right)\right] \\
& +\sum_{m \text { odd }} x(m)\left[\cos \left\{-\frac{\pi k}{2 N}+\frac{(m+1) \pi k}{N}\right\}+\sin \left\{-\frac{\pi k}{2 N}+\frac{(m+1) \pi k}{N}\right\}\right] \\
\text { or } \quad H(k) \operatorname{cas}\left(-\frac{\pi k}{2 N}\right) & =\sum_{m \text { even }} x(m)\left[\cos \frac{(2 m+1) \pi k}{2 N}+\sin \frac{(2 m+1) \pi k}{2 N}\right] \\
& +\sum_{m \text { odd }} x(m)\left[\cos \frac{(2 m+1) \pi k}{2 N}+\sin \frac{(2 m+1) \pi k}{2 N}\right]
\end{aligned}
$$

Replacing $k$ by $(N-k)$ in (13), we have

$$
H(N-k)=\sum_{m \text { even }} x(m) \operatorname{cas}\left[\frac{m \pi(N-k)}{N}\right]+\sum_{m \text { odd }} x(m) \operatorname{cas}\left[-\frac{(m+1) \pi(N-k)}{N}\right]
$$

Multiplying (17) with $\left[-\operatorname{cas}\left(\frac{\pi k}{2 N}\right)\right]$, we obtain

$$
\begin{aligned}
-H(N-k) \operatorname{cas}\left(\frac{\pi k}{2 N}\right)= & -\sum_{m \text { even }} x(m) \operatorname{cas}\left[\frac{m \pi(N-k)}{N}\right] \operatorname{cas}\left(\frac{\pi k}{2 N}\right) \\
& -\sum_{m \text { odd }} x(m) \operatorname{cas}\left[-\frac{(m+1) \pi(N-k)}{N}\right] \operatorname{cas}\left(\frac{\pi k}{2 N}\right) \\
& \text { for } k=0,1,2, \ldots, N-1
\end{aligned}
$$

Using the identity (15) in RHS of (18), we have

$$
\begin{aligned}
-H(N-k) \operatorname{cas}\left(\frac{\pi k}{2 N}\right)= & -\sum_{m \text { even }} x(m)\left[\cos \left\{\frac{m \pi(N-k)}{N}-\frac{\pi k}{2 N}\right\}+\sin \left\{\frac{m \pi(N-k)}{N}-\frac{\pi k}{2 N}\right\}\right] \\
& -\sum_{\text {modd }} x(m)\left[\cos \left\{\frac{\pi k}{2 N}+\frac{(m+1) \pi(N-k)}{N}\right\}+\sin \left\{\frac{\pi k}{2 N}+\frac{(m+1) \pi(N-k)}{N}\right\}\right] \\
= & -\sum_{m \text { even }} x(m)\left[\cos \left\{m \pi-\frac{(2 m+1) \pi k}{2 N}\right\}+\sin \left\{m \pi-\frac{(2 m+1) \pi k}{2 N}\right\}\right] \\
& -\sum_{m \text { odd }} x(m)\left[\cos \left\{(m+1) \pi-\frac{(2 m+1) \pi k}{2 N}\right\}+\sin \left\{(m+1) \pi-\frac{(2 m+1) \pi k}{2 N}\right\}\right] \\
= & -\sum_{m \text { even }} x(m)\left[\cos \left\{\frac{(2 m+1) \pi k}{2 N}\right\}-\sin \left\{\frac{(2 m+1) \pi k}{2 N}\right\}\right] \\
& -\sum_{m \text { odd }} x(m)\left[\cos \left\{\frac{(2 m+1) \pi k}{2 N}\right\}-\sin \left\{\frac{(2 m+1) \pi k}{2 N}\right\}\right] \\
\text { or }-H(N-k) \operatorname{cas}\left(\frac{\pi k}{2 N}\right) & =\sum_{m \text { even }} x(m)\left[-\cos \left\{\frac{(2 m+1) \pi k}{2 N}\right\}+\sin \left\{\frac{(2 m+1) \pi k}{2 N}\right\}\right] \\
& +\sum_{m \text { odd }} x(m)\left[-\cos \left\{\frac{(2 m+1) \pi k}{2 N}\right\}+\sin \left\{\frac{(2 m+1) \pi k}{2 N}\right\}\right]
\end{aligned}
$$

Adding (16) and (19), the cosine functions cancel and we get

$$
\begin{gathered}
H(k) \operatorname{cas}\left(-\frac{\pi k}{2 N}\right)-H(N-k) \operatorname{cas}\left(\frac{\pi k}{2 N}\right)=2\left[\sum_{m \text { even }} x(m) \sin \left\{\frac{(2 m+1) \pi k}{2 N}\right\}+\sum_{m \text { odd }} x(m) \sin \left\{\frac{(2 m+1) \pi k}{2 N}\right\}\right] \\
\text { or } \frac{1}{2}\left[H(k) \operatorname{cas}\left(-\frac{\pi k}{2 N}\right)-H(N-k) \operatorname{cas}\left(\frac{\pi k}{2 N}\right)\right]=\sum_{m=0}^{N-1} x(m) \sin \left[\frac{k(2 m+1) \pi}{2 N}\right] \\
\quad \text { for } k=0,1,2, \ldots, N-1
\end{gathered}
$$


According to (2), the RHS of above expression represents the type-II discrete sine transform $Y(k)$. Hence, we have

$$
\begin{gathered}
Y(k)=\frac{1}{2}\left[H(k) \operatorname{cas}\left(-\frac{\pi k}{2 N}\right)-H(N-k) \operatorname{cas}\left(\frac{\pi k}{2 N}\right)\right] \\
\quad \text { for } k=0,1,2, \ldots, N-1
\end{gathered}
$$

Since DST is given for $k=1,2, \ldots, N$, the $(N-1)$ output components $Y(k)$ of DST can be realized from DHT using (21).

Taking $\alpha=\frac{\pi k}{2 N}$, the above expression (21) can be written as

$$
\begin{aligned}
Y(k)= & \frac{1}{2}[H(k) \operatorname{cas}(-\alpha)-H(N-k) \operatorname{cas} \alpha] \\
= & \frac{1}{2}[H(k)(\cos \alpha-\sin \alpha)-H(N-k)(\cos \alpha+\sin \alpha)] \\
\Rightarrow Y(k)= & {[H(k)-H(N-k)]\left(\frac{1}{2} \cos \alpha\right)+[H(k)+H(N-k)]\left(-\frac{1}{2} \sin \alpha\right) } \\
& \text { for } k=1,2, \ldots, N-1
\end{aligned}
$$

The above expression gives the relation between type-II DST and type-I DHT. The $(N-1)$ output components of DST-II can be realized from DHT-I using (22). The data flow diagram for computation of DST-II from DHT-I using (22) is shown in Fig.1.

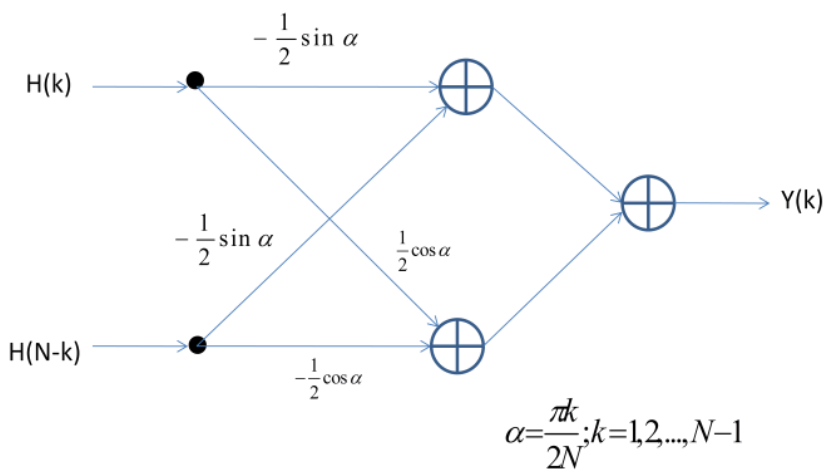

Fig.1. Data flow diagram for computation of DST-II from DHT-I.

\section{CONCLUSION}

In this paper, an expression for finding DSTII from DHT-I for even transform length $N$ has been derived. The $(N-1)$ output components of DST can be realized from DHT by this method. The data flow diagram for realization DST-II from DHT-I is shown.

\section{REFERENCES}

[1] A.K. Jain, "A fast Karhunen-Loeve transform for a class of random processes", IEEE Trans. Commun., vol. COM-24, pp.1023-1029, September 1976.

[2] H.B. Kekre and J.K. Solanka, “ Comparative performance of various trigonometric unitary transforms for transform image coding”, Int. J. Electron., vol. 44,pp.305315,1978 .

[3] A.K. Jain, "A sinusoidal family of unitary transforms", IEEE Trans. Patt. Anal. Machine Intell., vol. PAMI-I, pp.356-365, September 1979.

[4] Z. Wang and B. Hunt, "The discrete W transform", Applied Math Computat., vol. 16, pp.19- 48,January 1985.

[5] S. Cheng, "Application of the sine transform method in time of flight positron emission image reconstruction algorithms", IEEE
Trans. BIOMED. Eng., vol. BME-32, pp.185-192, March 1985.

[6] K. Rose, A. Heiman, and I. Dinstein, “ DCT/DST alternate transform image coding", Proc. GLOBECOM 87, vol. I,pp.426-430, November 1987.

[7] J.L. Wang and Z.Q. Ding, "Discrete sine transform domain LMS adaptive filtering", Proc. Int. Conf. Acoust., Speech, Signal Processing,pp.260-263, 1985.

[8] Z. Wang and L. Wang, "Interpolation using the fast discrete sine transform", Signal Processing, vol. 26, pp.131-137, 1992.

[9] A. K. Jain, Fundamentals of Digital Image Processing, Englewood Cliffs, NJ: Prentice Hall, 1989.

[10] P. Yip and K.R. Rao, "On the computation and the effectiveness of discrete sine transform", Comput. Electron., vol. 7,pp.45$55,1980$.

[11] R. N. Bracewell, "Discrete Hartley transform," J. Opt. Soc. Amer., vol. 73, no. 12, pp. 1832-1835, 1983.

[12] R. N. Bracewell, "The fast Hartley transform," Proc. IEEE, vol. 72, pp. 10101018, 1984. 
[13] R. N. Bracewell, The Fourier Transform and its Applications, $3^{\text {rd }}$ ed., New York: McGraw-Hill, 2000.

[14] L. -Z. Cheng, L. Tong, and Z. -R. Jiang, "Real transform algorithm for computing discrete circula deconvolution," in Proc. $3^{\text {rd }}$ IEEE Int. Conf. Signal Process., vol. 1, pp. 166-169, Oct. 1996.

[15] G. S. Maharana and P. K. Meher, "Algorithm for efficient interpolation of real-valued signals using discrete Hartley transform," Comp. Elect. Eng., vol. 23, no. 2, pp. 129-134, Mar. 1997.

[16] K. S. Tzou and T. R. Hsing, "A study of discreteHartley transform for image compression application," in Proc. SPIE Int. Soc. Opt. Eng., vol. 534, Jan. 1985.

[17] C. H. Paik and M. D. Fox, "Fast Hartley transform for image processing," IEEE Trans. Med. Imag., vol. 7, no. 6, pp. 149153, Jun.1988.

[18] J. -L. Wu and J. Shiu, "Discrete Hartley transform in error control coding," IEEE Trans. Signal Process., vol. 39, no. 10, pp. 2356-2359, Oct. 1991.

[19] P. K. Meher and G. Panda, "UnconstrainedHartley-domain least mean square adaptive filter," IEEE Trans. Circuits Syst. II, Analog Digit. Signal Process., vol. 40, no. 9, pp. 582-585, Sep. 1993.

[20] C. -L. Wang, C. -H. Chang, J. L. Fan, and J. M. Cioffi, "Discrete Hartley transform based multicarrier modulation," in Proc. IEEE Int. Conf. Acoust., Speech, Signal Process. (ICASSP'00), vol. 5, Jun. 2000, pp. 25132516.

[21] R. N. Bracewell, "Aspects of the Hartley transform," Proc. IEEE, vol. 82, no. 3, pp. 381-387, Mar. 1994.

[22] C. -L. Wang, C. -H. Chang, "A DHT-based FFT/IFFT processor for VDSL transceivers," in Proc. IEEE Int. Conf. Acoust., Speech, Signal Process. (ICASSP'01), vol. 2, May 2001, pp. 12131216.

[23] S. Bouguezel, M. O. Ahmad, and M. N. S. Swamy, "A New split-radix FHT algorithm for length $-q^{*} 2^{\mathrm{m}}$," IEEE Trans. Circuits Syst. I, Reg. Papers, vol. 51, no. 10, pp. 2031-2043, Oct. 2004.

[24] P. K. Meher, T. Srikanthan, and J. C. Patra, "Scalable and modular memory-based systolicarchitectures for discrete Hartley transform," IEEE Trans. Circuits Syst. I, Reg. Papers, vol. 53, no. 5, pp. 1065-1077, May 2006.

[25] P. K. Meher, J. C. Patra, and M. N. S. Swamy, "High-throughput memory-based architecture for DHT using a new convolutional formulation," IEEE Trans. Circuits Syst. II, Exp. Briefs, vol. 54, no. 7, pp. 606-610, Jul. 2007. 